\title{
Dealing with Music in Intelligent Ways
}

\author{
Gerhard Widmer ${ }^{1,2}$ \\ 1 Department of Computational Perception, Johannes Kepler University Linz \\ 2 Austrian Research Institute for Artificial Intelligence, Vienna, Austria \\ gerhard.widmer@jku. at \\ http://www.cp.jku.at/people/widmer
}

\begin{abstract}
Music is not just a product of human creativity and a uniquely human means of expression, it is also a commodity of great commercial relevance. The rapid digitisation of the music market, with the global availability of ever larger amounts of music, is creating a need for musically intelligent computer systems, and lots of opportunities for exciting research.

The presentation gives an impression of latest research in the field of intelligent music processing and music information retrieval. Based (mostly) on our own recent work, I discuss what it means for a computer to be musically intelligent, describe some of the techniques that are being developed, and demonstrate how entirely new musical interfaces and devices become possible with such methods - devices that, in effect, will change the way we listen to, and interact with, music.
\end{abstract}

